\title{
EVALUACIÓN DEL RIESGO ACUÁTICO DE SIETE PRODUCTOS FARMACÉUTICOS SOBRE Daphnia magna
}

\section{AQUATIC RISK ASSESSMENT OF SEVEN PHARMACEUTICAL PRODUCTS ON Daphnia magna}

\author{
José Iannacone ${ }^{12}$ y Lorena Alvariño ${ }^{3}$
}

\begin{abstract}
Resumen
La evaluación ecotoxicológica de productos farmacéuticos en el ambiente acuático dulceacuícola es un área de investigación emergente a nivel global. El objetivo del presente trabajo fue evaluar el efecto ecotoxicológico agudo de siete productos farmacéuticos ampliamente usados en el Perú: cuatro antimicrobianos (Amoxicilina, Ciprofloxacino y Trimetoprima + Sulfametoxazol), dos analgésicos (Ibuprofeno y Paracetamol), y un sedativo (Diazepan) sobre la pulga del agua Daphnia magna Strauss, 1820 (Crustacea: Daphniidae), y su relación con las principales características fisicoquímicas y de relación de componentes de la molécula, y a partir de estos resultados evaluar su riesgo ambiental acuático. Las pruebas de toxicidad aguda se evaluaron con cinco concentraciones más un control o testigo, y con cuatro repeticiones, en un diseño BCA de $6 \times 4$. Se observó la siguiente secuencia de ecotoxicidad decreciente en términos de $\mathrm{CL}_{50}\left(\mathrm{mg} \cdot \mathrm{L}^{-1}\right)$ a $96 \mathrm{~h}$ de exposición sobre $D$. magna: diazepan $(17,1)>$ paracetamol $(62.3)>$ ibuprofeno (175) > ciprofloxacino (230.6) > trimetoprima (296) > sulfametoxazol (1480) > amoxicilina (6950). No se encontró relación entre el $\operatorname{LogCL}_{50}$ y la proporción de producto excretado, el $\log \mathrm{K}_{\mathrm{ow}}$ y la solubilidad de los fármacos empleados. La ecotoxicidad potencial indicó que el diazepan ocasionó el mayor riesgo en el ambiente acuático. En cambio a través del cociente de riesgo solo el paracetamol produjo riesgo en el ambiente acuático.
\end{abstract}

Palabras clave: Amoxicilina, Ciprofloxacino, Daphnia, Diazepan, Ibuprofeno, Paracetamol, Sulfametoxazol, Trimetropima

\begin{abstract}
Ecotoxicological assessment of pharmaceutical products in freshwater environments is an emergent research globally. The aim of this research was to evaluate acute ecotoxicological effects of seven pharmaceutical products widely used in Peru: four antimicrobians (Amoxicillin, Ciprofloxacin and Trimethoprim + Sulfamethoxazole), two analgesics (Ibuprofen and Paracetamol), and a sedative (Diazepan) on freshwater flea Daphnia magna Strauss, 1820 (Crustacea: Daphniidae). Relationships with the main physicochemical characteristics and molecule compounds were also assessed in order to evaluate environmental aquatic risks. Acute toxicity test were evaluated with five concentrations plus a control, with four repetitions, using a $6 \times 4$ CRB design. The following decreasing ecotoxicity sequence in terms of $\mathrm{LC}_{50}\left(\mathrm{mg} \cdot \mathrm{L}^{-1}\right)$ at 96 h of exposure on D. magna was observed: Diazepan (17.1) > Paracetamol (62.3) > Ibuprofen (175) > Ciprofloxacin (230.6) > Trimethoprim (296) > Sulfamethoxazole (1480) > Amoxicillin (6950). Relationships between LogLC $\mathrm{C}_{50}$ and excreted product proportion, $\log \mathrm{K}_{\mathrm{ow}}$ and solubility of pharmaceutical products were not found. Potential ecotoxicity indicated that Diazepan caused the highest aquatic risk. However, using the quotient risk only Paracetamol produced aquatic risk.
\end{abstract}

Key words: Amoxicillin, Ciprofloxacin, Daphnia, Diazepan, Ibuprofen, Paracetamol, Trimethoprim

\section{Introducción.}

Los productos farmacéuticos son sustancias que intentan curar, mitigar o prevenir en su aplicación en el cuerpo humano o animal, de enfermedades, sufrimiento, daño corporal y problemas patológicos. Los productos de cuidado personal y cosméticos (PPCPs) como las fragancias, champús y cosméticos en conjunto con los fármacos activos o emergentes debido a su uso intenso y extensivo ingresan al ambiente cada año, y estiman que su cantidad es similar al total de plaguicidas utilizados durante el mismo periodo (Filho et al., 2007).

El uso desenfrenado de medicamentos debido a su aporte continuo y persistencia, inclusive de sus productos de degradación está ocasionado muchos efectos no deseados en el ambiente acuático como la emergencia y transmisión de genes de resistencia a antibióticos, daño a las comunidades microbianas por los desinfectantes, variación en el ritmo de vida y en las relaciones tróficas por los anestésicos, reducción 
en la fertilidad y el cambio de la condición sexual por hormonas y efectos tóxicos-reproductivos por drogas citostáticas (Bila \& Dezotti, 2003; Filho et al., 2007; Kratz, 2008). Existe evidencia que muchos de estos productos son persistentes en el ambiente y pueden llegar a las aguas superficiales y subterráneas, produciendo impactos en los organismos acuáticos no destinatarios, en muchas formas que incluye la mortalidad, errores en la muda o eclosión, deformidades anatómicas, cambios subletales en el crecimiento de las plantas, cambios en las proporción sexual de los organismos superiores, modificaciones en los ciclos biogeoquímicos, etc. Muchas de estas sustancias son encontradas en concentraciones de $\mathrm{ug} \cdot \mathrm{L}^{-1} \mathrm{y} \mathrm{ng} \cdot \mathrm{L}^{-1}$ en desagües domésticos, en aguas superficiales y en el subsuelo (Bila \& Dezotti, 2003). Fármacos con diferentes estructuras, funciones y actividades como antibióticos, hormonas, anestésicos, antihipertensivos, antiulcerosos, antilipémicos, cardiovasculares, antidrepesivos, medios de contraste de rayos $\mathrm{X}$ y antiinflamatorios entre otros han sido detectados en diferentes matrices ambientales acuáticas (Bila \& Dezotti, 2003). La principal forma de eliminar estos residuos farmacéuticos es a través de una eficiente remoción durante el tratamiento de las aguas residuales (Jjemba, 2006; Filho et al., 2007).

Muchos de estos productos químicos farmacéuticos aplicados en medicina humana y veterinaria, son producidos $\mathrm{y}$ usados en grandes volúmenes a nivel mundial. Por ende, la evaluación de riesgos de estos productos farmacéuticos es un área de investigación emergente en el ámbito global (HallingSørensen et al., 1998; Daughton \& Ternes, 1999; Chapman, 2006; Jjemba, 2006). Una cantidad desconocida de estos componentes son evacuados al ambiente vía las aguas residuales o cuando las heces son usadas con residuos farmacéuticos para la fertilización de tierras agrícolas (Lützhoft et al., 1999; Backhaus et al., 2000; Kümmerer, 2003; Lalumera et al., 2004; Woodward, 2006). Diferentes estudios han demostrados que muchos fármacos y sus metabolitos están presentes en ambientes acuáticos en varias partes del mundo (Bila \& Dezotti, 2003).

Debido a su rol clave en la comunidad zooplanctónica, su facilidad de cultivo en condiciones de laboratorio, ser partenogenética, producción de un alto numero de progenie en corto tiempo y a su extensa distribución geográfica, Daphnia magna Strauss 1820 (Crustácea: Daphniidae) es una especie empleada ampliamente en ensayos ecotoxicológicos para evaluar sustancias químicas puras, aguas residuales domésticas e industriales, aguas superficiales o subterráneas, agua potable, y lixiviados entre otros (Castillo, 2004; Källqvist et al., 2006; Reynaldi et al., 2006; Tonkopii et al., 2008).

La investigación del efecto ecotoxicológico proporciona más solidez a la evaluación de la calidad de agua (Wong \& Pak, 2004). Por este motivo el uso de los bioensayos son extremadamente importantes para determinar los efectos de los fármacos (Park \& Choi, 2008). Por medio de estos estudios se establecen los criterios de calidad para la protección de la vida acuática, los que posteriormente, se usan para determinar los estándares de calidad ambiental para cada agente químico (Burton \& Nordstrom, 2004). Los parámetros de toxicidad más comúnmente empleados son la concentración letal media $\left(\mathrm{CL}_{50}\right)$, la concentración efectiva media $\left(\mathrm{CE}_{50}\right)$ y la concentración de inhibición media $\left(\mathrm{CI}_{50}\right)$.

Amoxicilina es una penicilina semisintética bactericida que inhibe la síntesis de la pared celular bacteriana. Su acción depende de su capacidad para alcanzar y unirse a las proteínas que ligan penicilinas localizadas en la membrana interna de la pared celular bacteriana. Actúa contra un amplio espectro de microorganismos, tanto gram-positivos y gramnegativos. Por esto se emplea a menudo como primer remedio en infecciones tanto en medicina humana como también en veterinaria. Se utiliza por vía oral o parenteral, aunque la forma parenteral (intramuscular o intravenosa) no está aprobada en todos los países (Calvo \& Martínez, 2009).

Ciprofloxacino es una fluoroquinolona bactericida que actúa intracelularmente inhibiendo la topoisomerasa II y la topoisomerasa IV. Los topoisomerasas son enzimas bacterianas esenciales que son catalizadores críticos en la duplicación, trascripción y reparación del ADN bacteriano. Es un antibiótico usado para tratar ciertas infecciones causadas por bacterias como la neumonía, gonorrea, diarrea infecciosa, fiebre tifoidea, carbunco por inhalación (después de la exposición a una fuente de contaminación), y las infecciones a los huesos, articulaciones, la piel y las vías urinarias (Calvo \& Martínez, 2009).

El diazepan es una benzodiazepina que actúa como depresor del sistema nervioso central produciendo, desde una leve sedación hasta hipnosis o coma dependiendo de la dosis. Puede ser administrada por vía oral, intramuscular, o intravenosa para la ansiólisis (incluyendo desórdenes de ansiedad y de pánico), actividad anticonvulsiva, sedación preoperatoria, desintoxicación alcohólica/delirium tremens, y relajación del músculo esquelético (Straub, 2008).

El ibuprofeno es un anti-inflamatorio no esteroideo que inhibe la actividad de la enzima ciclooxigenasa, dando lugar a una disminución de precursores de las prostaglandinas y de los tromboxanos a partir del ácido araquidónico. Es utilizado frecuentemente para el alivio sintomático del dolor de cabeza (cefalea), dolor dental, dolor muscular (mialgia), molestias de la menstruación (dismenorrea), síndrome febril, y dolor tras cirugía (postquirúrgico). También se usa para tratar cuadros inflamatorios, como los que se presentan en artritis, artritis 
reumatoide (AR) y artritis gotosa (Carabaño et al., 2005).

El paracetamol es un para-aminofenol, analgésico y antipirético efectivo, indicado para aliviar dolores ligeros o moderados y para reducir la fiebre. Actúa inhibiendo la síntesis de prostaglandinas, mediadores celulares responsables de la aparición del dolor. Es un medicamento con propiedades analgésicas, sin propiedades antiinflamatorias clínicamente significativas. En la actualidad es uno de los analgésicos más utilizados y no interactua con la gran mayoría de los medicamentos (Carabaño et al., 2005).

Trimetoprima es un trimetoxibenzilpirimidina antiséptico bacteriostático de amplio espectro. Es un análogo estructural del acido para-aminobenzoico (PABA) e inhibe competitivamente la dihidrofolato reductasa. Suele presentarse en combinación con el sulfametoxazol, cuya combinación recibe el nombre de cotrimoxazol. El sulfametoxazol es una sulfonamida eficaz empleada para el tratamiento sistémico de infecciones bacterianas en el ser humano (Calvo \& Martínez, 2009).

Estos fármacos aplicados con propósitos medicinales pueden alcanzar finalmente al medio dulceacuícola por rutas directas o indirectas (Kümmerer, 2003; Brain et al., 2008). Sin embargo, no se conoce adecuadamente las consecuencias ecológicas del ingreso de estas sustancias a los cuerpos agua. Por ende, la evaluación del efecto de estos compuestos en organismos acuáticos no destinatarios, como la pulga de agua $D$. magna, a través de una evaluación del riesgo ecológico permitirá tomar las medidas de mitigación necesarias para la protección de los ambientes acuáticos por productos farmacéuticos (Zuccato et al., 2006).

El objetivo del presente trabajo fue evaluar el efecto toxicológico agudo de siete productos farmacéuticos: amoxicilina, ciprofloxacino, trimetropima más sulfametoxazol, ibuprofeno, paracetamol y diazepan sobre la pulga del agua $D$. magna, y a partir de estos resultados evaluar el riesgo ambiental acuático de estos productos.

\section{Materiales y métodos.}

Los bioensayos toxicológicos agudos a $96 \mathrm{~h}$ de exposición de los siete productos farmacéuticos: amoxicilina, ciprofloxacino, diazepan, ibuprofeno, paracetamol y trimetoprima más sulfametoxazol sobre la pulga del agua $D$. magna se realizaron en la Facultad de Ciencias Biológicas, Universidad Ricardo Palma, Distrito de Santiago de Surco, Lima, Perú. Productos farmacéuticos.

Con relación al criterio de selección de seis medicamentos (amoxicilina, ciprofloxacino, diazepan, ibuprofeno, paracetamol, trimetoprima + sulfametoxazol, usado estos dos últimos en combinación) se tomó en consideración lo reportado por Jjemba (2006), quien señala que estos siete productos farmacéuticos presentaban referencias con relación a sus propiedades fisicoquímicas, biodisponibilidad, proporción o porcentaje excretado, y cantidades detectadas en el ambiente con el fin de proporcionar información para la interpretación del riesgo en el ambiente dulceacuícola, a partir de los resultados obtenidos en los bioensayos. En adición se incluyó como criterio que los seis, son fármacos ampliamente usados en el Perú. Para los siete productos farmacéuticos o medicamentos seleccionados se emplearon cinco concentraciones nominales a partir de sus respectivos ingredientes activos de los productos comerciales para ser usados en los ensayos ecotoxicológicos con $D$. magna. Se empleó para la amoxicilina (Amoxil®) pastillas 500 mg, para ciprofloxacino (Ciproflox ${ }^{\circledR}$ ) pastillas 500 $\mathrm{mg}$, para diazepan (Valium ${ }^{\circledR}$ ) pastillas $10 \mathrm{mg}$, para ibuprofeno (Dolomaxl®) pastillas $400 \mathrm{mg}$, para paracetamol (Panadol $\left.{ }^{\circledR}\right)$ y finalmente para trimetoprima y sulfametoxazol (cotrimoxazol) (Bactrim ${ }^{\circledR}$ ) pastillas de 160 y $800 \quad$ mg, respectivamente. Las concentraciones ha emplear fueron determinadas empleando un factor de dilución de 0.5 usando agua embotellada (Cielo $®)$, tomando en consideración los criterios de Jjemba (2006). Para el diazepan se usó etanol al 96\% para disolver la sustancia química.

Para cada uno de los productos farmacéuticos empleados se obtuvo su fórmula química, su número de registro CAS, su uso común, su peso molecular, su $\operatorname{logK}_{\text {ow }}$ (Coeficiente octanol agua), su pKa (Constante ácido Base), su CAE o PEC (Concentración Ambiental Esperada en $\mathrm{mg} \mathrm{L}^{-1}$, la proporción de producto excretado (\%), su solubilidad, su relación $\mathrm{C} / \mathrm{O}$ e H/O (Tablas 1 y 2) (Kummerer, 2004; Jjemba, 2006; Cooper et al., 2008).

Daphnia magna.

Hembras adultas se obtuvieron de un acuario del distrito de Lince, Lima, Perú, y fueron llevadas al laboratorio en recipientes plásticos de $2 \mathrm{~L}$ de capacidad. Hembras partenogenéticas se colocaron en el medio nutritivo ADaM. La preparación del medio se realizó de la siguiente forma: $9.9 \mathrm{~g}$ de sales obtenidas por evaporación del agua de mar se adicionaron a $60 \mathrm{~L}$ de agua de grifo declorinada, reposada y hiperoxigenada durante $24 \mathrm{~h}$. Luego se agregó $138 \mathrm{~mL}$ de una solución de cloruro de calcio (117.6 $\left.\mathrm{g} \cdot \mathrm{L}^{-1}\right), 132 \mathrm{~mL}$ de una solución de bicarbonato de sodio $\left(25.2 \mathrm{~g} \cdot \mathrm{L}^{-1}\right)$ y $6 \mathrm{~mL}$ de una solución de Oxido de Selenio $\left(0.07 \mathrm{~g} \mathrm{~L}^{-1}\right)$ (Klüttgen et al., 1994). Los cultivos parciales se mantuvieron a una temperatura de $21 \pm 2^{\circ} \mathrm{C}$ y a un fotoperiodo aproximadamente de 12:12. El oxígeno disuelto tuvo una concentración sobre $6 \mathrm{mg} \cdot \mathrm{L}^{-1}$ al ser determinado por el método de Winlkler (Castillo, 2004). Para el desarrollo de la prueba de toxicidad aguda con $D$. magna se emplearon cohortes de neonatos $(<24 \mathrm{~h}$ de nacidos). La duración total de la prueba fue de 96 h de 
exposición. Se empleó un factor de dilución de 0,5. A cada envase circular de $250 \mathrm{~mL}$ se procedió a agregar $100 \mathrm{~mL}$ de cada una de las concentraciones de las sustancias químicas empleadas, a los que se transfirieron diez neonatos de D. magna. Se usó la carencia de movilidad o la ausencia de ritmo cardiaco a $15 \mathrm{~s}$ de observación al microscopio estereoscopio como criterio de mortalidad. Antes de efectuar las lecturas se agitó los envases en forma circular para reactivar el movimiento de los organismos que se posaban inmóviles en el fondo (Castillo, 2004).

Diseño y análisis estadístico

Las pruebas de ecotoxicidad aguda fueron evaluadas en cinco concentraciones, más un control o testigo, y con cuatro repeticiones, en un diseño en bloque completamente aleatorizado (DBCA) de 6 x 4 . En todos los casos la eficacia de los tratamientos se determinó a través de un análisis de Varianza (ANDEVA) de dos vías, previa transformación de los porcentajes de mortalidad a raíz cuadrada del arcoseno, con el fin de ajustar los datos a la distribución normal. En el caso de diferencias significativas entre los tratamientos se realizó una Prueba de Significación DVS (Diferencia Verdaderamente Significativa) de Tukey. La CL L $_{50}$ y sus respectivos límites de confianza al $95 \%$, se calcularon usando el programa computarizado EPA Probit versión 1.5. Se determinó el valor de NOEC (Concentración de efectos no observables) a 96 h de exposición, como aquella concentración en la que no existen diferencias con el control. Se correlacionó la $\mathrm{CL}_{50}$ con los principales parámetros fisicoquímicos de cada uno de los productos farmaceúticos. La Relación agudo-crónico (RAE) se calculó como la proporción entre la $\mathrm{CL}_{50}$ a $96 \mathrm{~h}$ y el valor de NOEC a $96 \mathrm{~h}$. La ecotoxicidad potencial $(\mathrm{EP})=\mathrm{T} /(\mathrm{CAE} \times \mathrm{NOEC} 96 \mathrm{~h})$, donde $\mathrm{T}=$ Tiempo de residencia en el ambiente $\mathrm{o}$ biodisponibilidad considerando una descarga continua de 365 días, la concentración en el ambiente o degradabilidad (CAE) y finalmente el valor de NOEC a 96 h de exposición (Jjemba, 2006). En adición se riesgo de los siete productos en el ambiente acuático dulceacuícola (Halling-Sorensen et al., 2000). La caracterización del riesgo se estimó a partir del CR determinado de la relación de la evaluación de exposición PEC (concentración ambiental en el agua en $\mathrm{mg} \cdot \mathrm{L}^{-1}$ ) y del efecto de evaluación PNEC (concentración ambiental de toxicidad en agua en $\mathrm{mg} \cdot \mathrm{L}^{-1}$ usando el peor escenario sin degradación en el ambiente dulceacuícola). Los valores de PEC fueron obtenidos para amoxicilina, diazepan, ibuprofeno, sulfametoxazol y paracetamol de Kûmmerer (2004) y para ciprofloxacino y trimetoprima de HallingSørensen et al. (2000). Se tomaron en consideración todas las asunciones propuestas por Halling-Sórensen et al. (2000) para su determinación a partir de la siguiente fórmula: $\mathrm{PEC}=\mathrm{A} \times(100-\mathrm{R}) /(365 \mathrm{x} \mathrm{P} \times \mathrm{V}$ x $\mathrm{D} \times 100$ ) donde $\mathrm{A}=$ la cantidad empleada por año

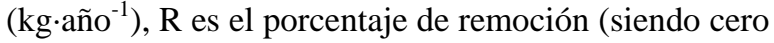
en las peores condiciones sin degradación), $\mathrm{V}$ es el volumen de desagüe per capita por día $\left(0.2 \mathrm{~m}^{3}\right)$. P es la población en millones que usa el producto farmacéutico, D es el factor de dilución de 10) y A es el tiempo de retención hidráulica igual a 10 h. Los valores de PNEC de cada producto farmacéutico se determinaron de la relación [ $\mathrm{CL}_{50}$ a 96 h de exposición para D. magna para cada uno de los siete productos farmacéutico/100] a partir de las guías de la OECD empleando un factor de seguridad de 100 (OECD, 1992).

\section{Resultados.}

La Tabla 1 nos indica la fórmula química, CAS, uso común, peso molecular, $\log \mathrm{K}_{\mathrm{ow}}, \mathrm{pKa}, \mathrm{CAE}$ o PEC, proporción del producto excretado y la solubilidad en agua de los siete productos farmacéuticos y productos de cuidado personal (PPCPs). Se observó la siguiente secuencia de ecotoxicidad decreciente en términos de $\mathrm{CL}_{50}\left(\mathrm{mg} \cdot \mathrm{L}^{-}\right.$ 1): diazepan (17.1) > paracetamol (62.3) >ibuprofeno (175) > ciprofloxacino (230) > trimetoprima $(296)>$ sulfametoxazol (1480) > amoxicilina (6950) (Tabla 2).

Tabla 1.- Principales características fisicoquímicas de productos farmacéuticos y productos de cuidado personal (PPCPs) empleados sobre Daphnia magna. pKA = constante acido base. $\mathrm{K}_{\mathrm{ow}}=$ coeficiente octanol agua. $\mathrm{CAE}=$ Concentración ambiental esperada. Prop-Exc. = Proporción excretado. Trimetroprima + sulfametoxazol $=$ cotrimoxazol.

\begin{tabular}{|c|c|c|c|c|c|c|c|c|c|}
\hline PPCPs & Formula química & CAS & uso común & $\begin{array}{c}\text { Peso } \\
\text { Molecular } \\
\end{array}$ & $\log \mathrm{K}_{\mathrm{ow}}$ & pka & $\begin{array}{c}\mathrm{CAE} \\
\left(\mathrm{ug} \cdot \mathrm{L}^{-1}\right) \\
\end{array}$ & $\begin{array}{c}\text { Prop.Exc. } \\
(\%)\end{array}$ & $\begin{array}{c}\text { Solubilidad } \\
\text { en agua }\end{array}$ \\
\hline Amoxicilina & $\mathrm{C}_{16} \mathrm{H}_{19} \mathrm{~N}_{3} \mathrm{O}_{53} \mathrm{H}_{2} \mathrm{O}$ & $61336-70-7$ & antibiótico & 365.4 & 0.87 & 2.4 & 3.13 & $80-90$ & 4000 \\
\hline Ciprofloxacino & $\mathrm{C}_{17} \mathrm{H}_{18} \mathrm{FN}_{3} \mathrm{O}_{3} \mathrm{HCl}$ & 85721-33-1 & antibiótico & 331.3 & 1.32 & 5.9 & 2.44 & 83.7 & 10000 \\
\hline Diazepan & $\mathrm{C}_{16} \mathrm{H}_{13} \mathrm{ClN}_{2} \mathrm{O}$ & $439-14-5$ & sedativo & 284.7 & 2.98 & 3.4 & 0.12 & 1 & 10 \\
\hline Ibuprofeno & $\mathrm{C}_{13} \mathrm{H}_{18} \mathrm{O}_{2}$ & $15687-271$ & analgésico & 206.3 & 3.14 & 5.2 & 18.92 & $1-8$ & 10 \\
\hline Paracetamol & $\mathrm{C}_{8} \mathrm{H}_{9} \mathrm{NO}_{2}$ & $103-90-2$ & analgésico & 151.2 & 0.89 & 9.4 & 15.69 & $<5$ & 100 \\
\hline Trimetoprima & $\mathrm{C}_{14} \mathrm{H}_{18} \mathrm{~N}_{4} \mathrm{O}_{3}$ & 738705 & antibacteriano & 290.3 & 1.43 & 6.6 & 1.26 & 60 & 400 \\
\hline Sulfametoxazol & $\mathrm{C}_{10} \mathrm{H}_{11} \mathrm{~N}_{3} \mathrm{O}_{3} \mathrm{~S}$ & $723-46-6$ & antibacteriano & 253.3 & 0.86 & 6.0 & 0.15 & 15 & 10 \\
\hline
\end{tabular}

Extraida de Kummerer (2004), Jjemba (2006) y Cooper et al. (2008).

empleó el cociente de riesgo (CR) para determinar el

La Tabla 3 nos indica el porcentaje de mortalidad 
en $D$. magna por acción de cada una de las cinco concentraciones $\left(\mathrm{mg} \cdot \mathrm{L}^{-1}\right)$ de siete productos farmacéuticos y productos de cuidado personal (PPCPs) a 96 h de exposición. De igual forma se señalan para todos los casos los valores del estadístico de Fisher (F) según el ANDEVA y los valores de significancia estadística $<0.001$. No se observaron diferencias significativas entre las repeticiones para relación a la proporción $\mathrm{C} / \mathrm{O}$ se encontró la siguiente secuencia en orden decreciente: diazepan > ibuprofeno $>$ ciprofloxacino $>$ trimetoprima $>$ paracetamol $>$ sulfametoxazol $>$ amoxicilina. Para la relación $\mathrm{H} / \mathrm{O}$ se vio la siguiente secuencia similar en orden decreciente: diazepan $>$ ibuprofeno $>$ ciprofloxacino $=$ trimetoprima $>$ paracetamol $>$ sulfametoxazol $>$ amoxicilina (Tabla 2). El RAC mostró la siguiente 1a:amoxicilina

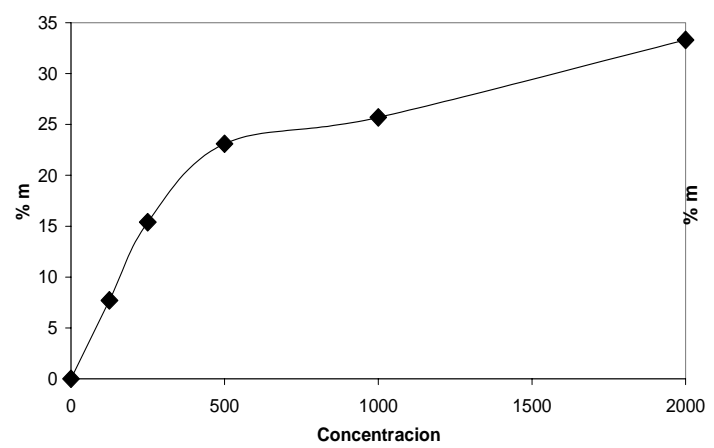

1c: diazepam

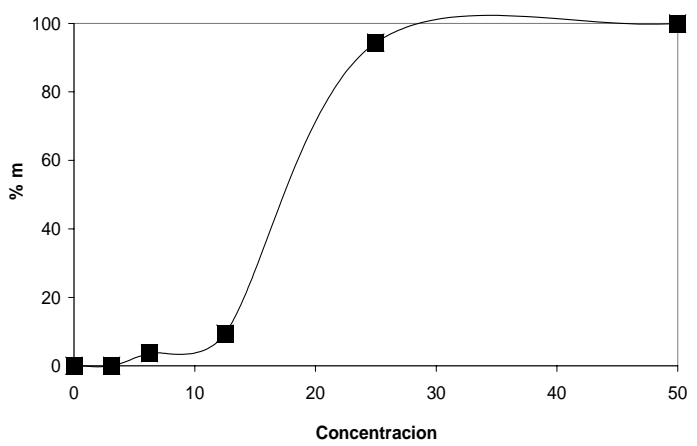

1e:paracetamol

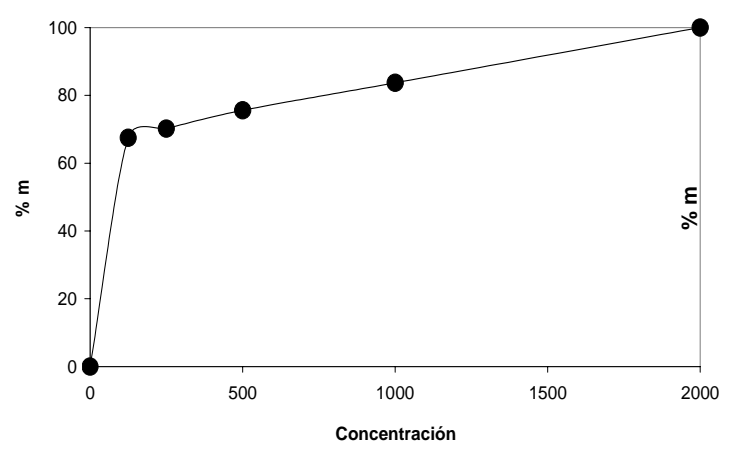

1b:ciprofloxacina

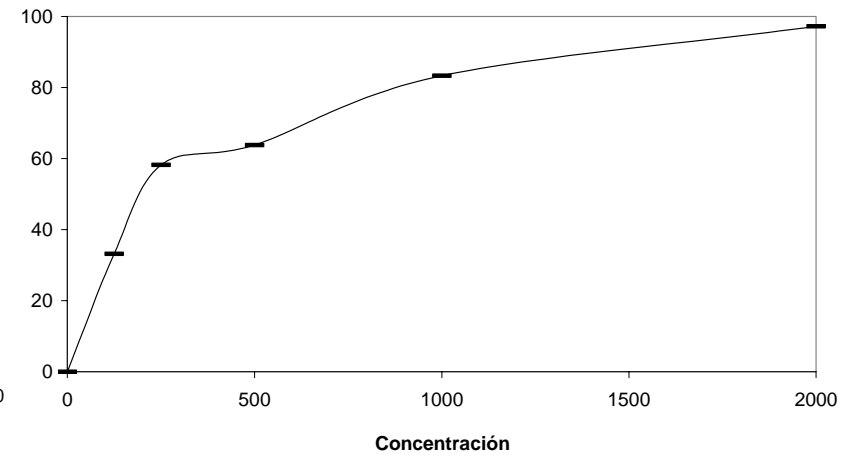

1d:ibuprofeno
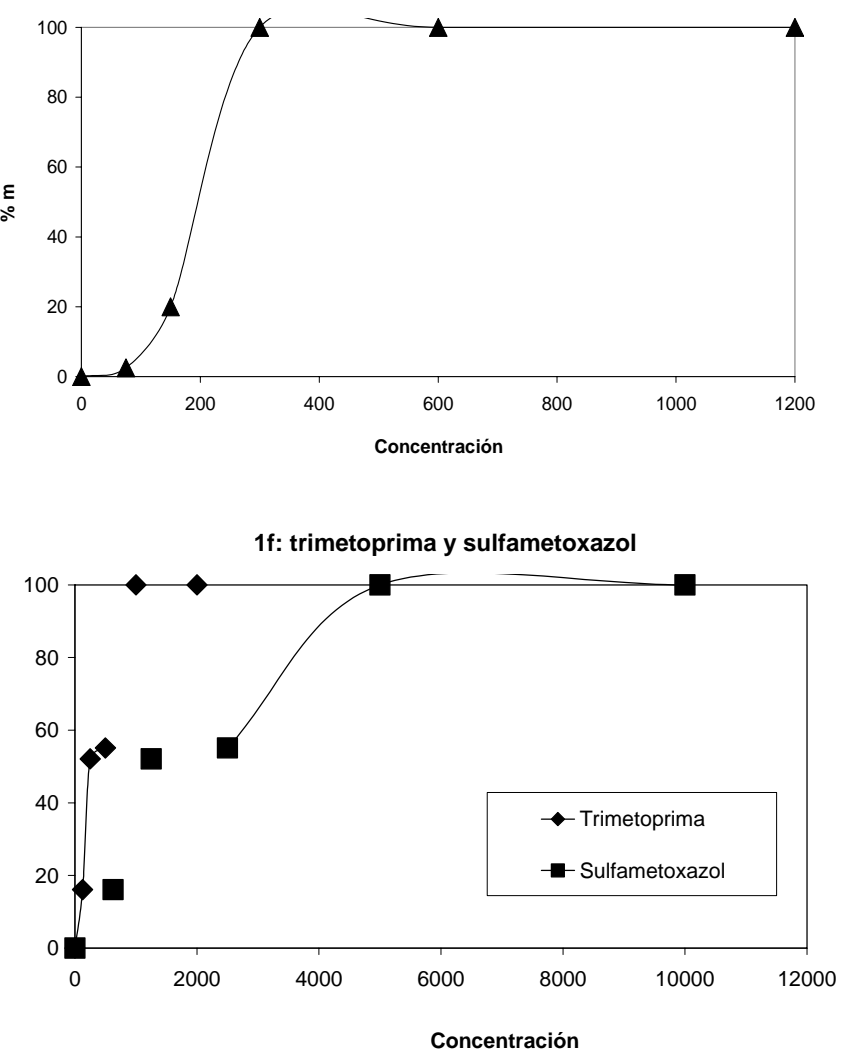

Figura 1. Curvas de concentración $\left(\mathrm{mg} \cdot \mathrm{L}^{-1}\right)$ versus mortalidad de siete productos farmacéuticos sobre Daphnia magna.

los bioensayos con D. magna con cada producto farmacéutico. La fig. 1 nos muestras las curvas de concentración de los seis PPCPs versus el porcentaje de mortalidad de D. magna a 96 h de exposición. Con secuencia en orden decreciente: amoxicilina > trimetoprima $=$ sulfametoxazol $>$ ciprofloxacino $>$ ibuprofeno $>$ diazepan $>$ paracetamol. La EP nos indicó el siguiente ordenamiento descendente: 
diazepan > sulfametoxazol $>$ trimetoprima $>$ ciprofloxacino $>$ paracetamol $>$ ibuprofeno $>$ amoxicilina (Tabla 2). La Tabla 4 nos muestra que solo el paracetamol provocó riesgo en el ambiente acuático al ser su cociente de riesgo mayor a 1 .

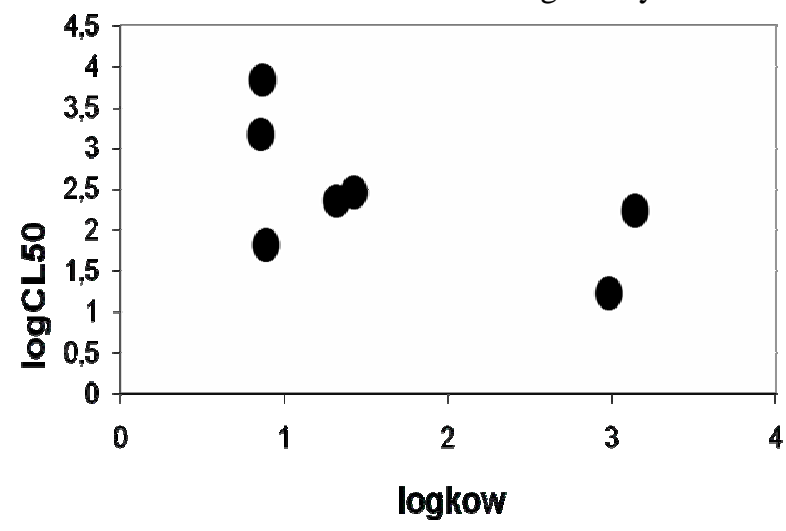

Figura 2. Relación entre el $\log \mathrm{CL}_{50}$ para siete productos farmacéuticos sobre Daphnia magna y su logKow.

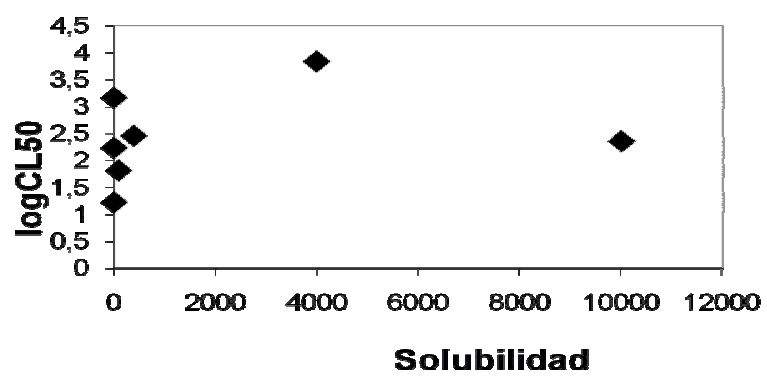

Figura 3. Relación entre $\log \mathrm{CL}_{50}$ para siete productos farmacéúticos sobre Daphnia magna y su solubilidad.

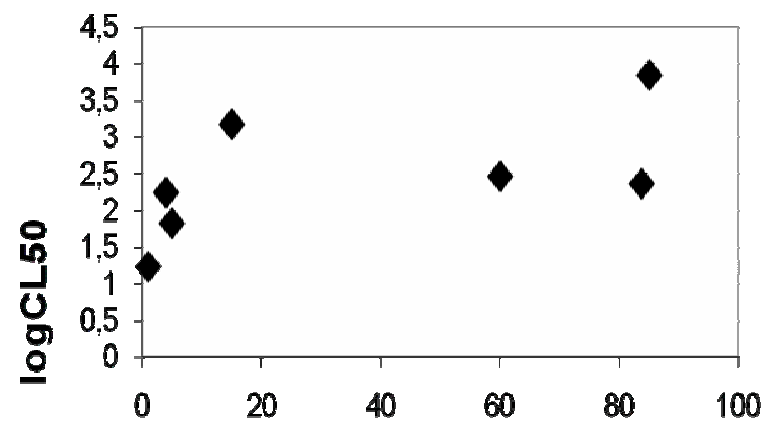

Proporción Excretado (\%)

Figura 4. Relación entre $\log \mathrm{CL}_{50}$ de siete productos farmacéuticos sobre Daphnia magna y la proporción de producto excretado (\%).

No se observaron correlaciones significativas entre el $\log \mathrm{CL}_{50}$ de los PPCPs y sus logKow ( $\mathrm{r}=$ 0.60 y $\mathrm{P}=0.15$ ) (Figura 2). De igual forma se vio una ausencia de correlación entre el logCL $\mathrm{L}_{50}$ de los PPCPs y la solubilidad $(\mathrm{r}=0.24$ y $\mathrm{P}=0.60)$ (Figura 3). Finalmente, se encontró una ausencia de correlación significativa entre la proporción excretada (\%) de PPCPs y el $\log \mathrm{CL}_{50}(\mathrm{r}=0.59$ y $\mathrm{P}=0.15)$ (Figura 4$)$.

\section{Discusión.}

La existencia muy elevada de fármacos activos en el ambiente acuático estimula ha realizar mayores estudios de impacto ambiental (Brain et al., 2008). Sin embargo los estudios ecotoxicológicos levantados por muchos investigadores para identificar fármacos potencialmente peligrosos para el medio ambiente son aún insuficientes (Bila \& Dezotti, 2003). Algunos investigadores han analizado el riesgo potencial de algunos fármacos en el medio ambiente a través de la biodegradabilidad, del destino ambiental y de las pruebas de toxicidad con organismos acuáticos (Castiglioni et al., 2004; Brain et al., 2008; Choi et al., 2008; Park \& Choi, 2008).

El diazepan fue la sustancia química que presentó la mayor ecotoxicidad potencial y la mayor $\mathrm{CL}_{50}$ a 96 h $\left(17.1 \mathrm{mg} \cdot \mathrm{L}^{-1}\right)$ sobre $D$. magna en comparación con los otros productos farmacéuticos (Tabla 2). El valor de $\mathrm{CL}_{50}$ obtenido es bastante similar a lo registrado en la literatura por otros autores, de 4.3 a $14 \mathrm{mg} \cdot \mathrm{L}^{-1}$ a 24 h de exposición. Los peces como Oncorhynkus mykiss (Walbaum 1792) a 96 h de exposición presentaron menor sensibilidad al diazepan $\left(84 \mathrm{mg} \cdot \mathrm{L}^{-1}\right)$ y las algas como Scenedesmus subspicatus Chodat,1926 y Synechococcus leopoliensis (Raciborski, 1849) Komarek, 1932 presentaron valores similar al de $D$. magna $\left(\mathrm{CI}_{50}\right.$ de 3.11 y $11.9 \mathrm{mg} \cdot \mathrm{L}^{-1}$, respectivamente) (Straub, 2008).

El sulfametoxazol y la trimetoprima que fueron evaluados como una mezcla presentaron en secuencia al diazepan una alta ecotoxicidad potencial sobre $D$. magna (Tabla 2). La trimetoprima y el sulfametoxazol al ser evaluados en comparación con otros nueve antibióticos, se encontraron entre los más tóxicos sobre Vibrio fischeri (Beijerinck 1889) Lehmann \& Neumann 1896, D. magna, Moina macrocopa (Straus 1820) y Oryzias latipes (Temminck and Schlegel, 1846) (Park \& Choi, 2008; De Liguoro et al., 2009). Algunos investigadores al emplear el procedimiento del CR encontraron que el sulfametoxazol y la amoxicilina presentan valores que exceden la unidad y presentan riesgo potencial ecológico en el ambiente dulceacuícola (Choi et al., 2008; Siemens et al., 2008). Sin embargo, nuestros resultados con $D$. magna con estas dos sustancias mostraron ausencia de riesgo ambiental según el CR (Tabla 3). Yang et al. (2008) han encontrado al evaluar la $\mathrm{CI}_{50}$ en 12 agentes antibacterianos sobre la microalga Pseudokirchneriella subcapitata (Hindak 1990) que el ciprofloxacino y la trimetoprima presentaron poca toxicidad. De 10 productos farmacéuticos en Korea, una evaluación de riesgos indicó que el sulfametoxazol presentó un CR de 6.3 empleando $D$. 
magna, V. fischeri y O. latipes (Kim et al., 2007).

En el caso del ibuprofeno se han encontrado

Tabla 2.- Principales características toxicológicas y de relación de componentes de productos farmacéuticos y productos de cuidado personal (PPCPs) empleados sobre Daphnia magna. RAC = Relación agudo crónico. $\mathrm{EP}=$ Ecotoxicidad Potencial. Trimetroprima + sulfametoxazol $=$ cotrimoxazol.

\begin{tabular}{cccccc}
\hline PPCPs & $\mathrm{CL}_{50}\left(\mathrm{mg} \cdot \mathrm{L}^{-1}\right)$ & Relación $\mathrm{C} / \mathrm{O}$ & Relación $\mathrm{H} / \mathrm{O}$ & $\mathrm{RAC}$ & $\mathrm{EP}$ \\
\hline Amoxicilina & 6950 & 2.7 & 3.1 & 27.8 & $4.66 \times 10^{-6}$ \\
Ciprofloxacino & 230.6 & 5.2 & 6 & 1.84 & 0.001196 \\
Diazepan & 17.1 & 16 & 13 & 0.68 & 0.12 \\
Ibuprofeno & 175 & 6.5 & 9 & 1.16 & $1.28 \times 10^{-4}$ \\
Paracetamol & 62.3 & 4 & 4.5 & 0.53 & $1.86 \times 10^{-4}$ \\
Trimetoprima & 296 & 4.8 & 6 & 2.36 & 0.0023 \\
Sulfametoxazol & 1480 & 3.3 & 3.6 & 2.36 & 0.0038 \\
\hline
\end{tabular}

paracetamol a valores de $2 \mathrm{mg} \cdot \mathrm{L}^{-1}$ en el zooplancton y en los protozoos, y para los rotíferos en $8 \mathrm{mg} \cdot \mathrm{L}^{-1}$ a 48 $\mathrm{h}$ de exposición.

El ciprofloxacino usado sobre D. magna produjo un valor de $\mathrm{CL}_{50}$ a $96 \mathrm{~h}$ de $230.6 \mathrm{mg} \cdot \mathrm{L}^{-1}$. El ciprofloxacino no ha ocasionado efectos en el anfípodo detritívoro Gammarus spp. y en las larvas del trichóptero Lepidostoma liba Ross 1941. Sin embargo, si se observaron efectos del ciprofloxacino en las

Tabla 3.- Porcentajes de mortalidad en Daphnia magna por acción de cada una de las cinco concentraciones $\left(\mathrm{mg} \cdot \mathrm{L}^{-1}\right)$ de siete productos farmacéuticos y productos de cuidado personal (PPCPs) a $96 \mathrm{~h}$ de exposición.

\begin{tabular}{|c|c|c|c|c|c|c|c|c|c|c|c|c|c|}
\hline \multicolumn{2}{|c|}{ Amoxicilina } & \multicolumn{2}{|c|}{ Ciprofloxacino } & \multicolumn{2}{|c|}{ Diazepan } & \multicolumn{2}{|c|}{ Ibuprofeno } & \multicolumn{2}{|c|}{ Paracetamol } & \multicolumn{2}{|c|}{ Trimetoprima } & \multicolumn{2}{|c|}{ Sulfametoxazol } \\
\hline Conc. & $\% \mathrm{~m}$ & Conc. & $\% \mathrm{~m}$ & Conc. & $\% \mathrm{~m}$ & Conc. & $\% \mathrm{~m}$ & Conc. & $\% \mathrm{~m}$ & Conc. & $\% \mathrm{~m}$ & Conc. & $\% \mathrm{~m}$ \\
\hline 0 & $0 \mathrm{a}$ & 0 & $0 \mathrm{a}$ & 0 & $0 \mathrm{a}$ & 0 & $0 \mathrm{a}$ & 0 & 0a & 0 & 0a & 0 & $0 \mathrm{a}$ \\
\hline 125 & $7.7 \mathrm{a}$ & 125 & $33.2 b$ & 3.12 & $0 \mathrm{a}$ & 75 & $2.5 \mathrm{a}$ & 125 & $67.5 b$ & 125 & $16.1 b$ & 625 & $16.1 \mathrm{~b}$ \\
\hline 250 & $15.4 \mathrm{ab}$ & 250 & $58.2 \mathrm{c}$ & 6.25 & $3.7 \mathrm{a}$ & 150 & $20 \mathrm{~b}$ & 250 & $70.2 b$ & 250 & 52.1c & 1250 & $52.1 \mathrm{c}$ \\
\hline 500 & $23,1 b$ & 500 & $63.8 c$ & 12.5 & $9.4 \mathrm{a}$ & 300 & $100 \mathrm{c}$ & 500 & $75.6 b$ & 500 & $55.1 \mathrm{c}$ & 2500 & $55.1 c$ \\
\hline 1000 & $25.7 \mathrm{bc}$ & 1000 & 83.3d & 25 & $94.4 \mathrm{~b}$ & 600 & $100 \mathrm{c}$ & 1000 & $83.7 b$ & 1000 & $100 d$ & 5000 & $100 d$ \\
\hline 2000 & 33.3c & 2000 & $97.2 \mathrm{~d}$ & 50 & $100 \mathrm{~b}$ & 1200 & $100 c$ & 2000 & $100 c$ & 2000 & $100 d$ & 10000 & $100 d$ \\
\hline $\mathrm{F}$ & 42.1 & & 91.8 & & 81.1 & & 84.3 & & 88.7 & & 92.2 & & 89.5 \\
\hline Sig. & $<0.001$ & & $<0.001$ & & $<0.001$ & & $<0.001$ & & $<0.001$ & & $<0.001$ & & $<0.001$ \\
\hline
\end{tabular}

Conc. = Concentración. \% $\mathrm{m}=$ porcentaje de mortalidad. F= Estadístico de Fisher según el análisis de Varianza. Sig. = significancia. Letras minúsculas iguales en una misma columna señalan que los promedios de mortalidad son estadísticamente iguales según la prueba de Tukey $(P>0.05)$.

valores de $\mathrm{CL}_{50}$ a $96 \mathrm{~h}\left(175 \mathrm{mg} \cdot \mathrm{L}^{-1}\right)$ sobre $D$. magna.

Registros en la literatura con D. magna a $48 \mathrm{~h}$ mostró

un valor de $\mathrm{CL}_{50}$ de $55,6 \mathrm{mg} \cdot \mathrm{L}^{-1}$. Un análisis comparativo ha mostrado en Lepomis macrochirus Rafinesque 1819 a $\quad 96 \quad$ h $\quad$ y $\quad P$. subcapitata (=Selenastrum capricornutum Printz of Skulberg, 1964) valores de $\mathrm{CE}_{50}$ de $173 \mathrm{y}>30 \mathrm{mg} \cdot \mathrm{L}^{-1}$. Otro autor como Heckmann (2007) han encontrado menores valores en $D$. magna de $\mathrm{CE}_{50}$ a $48 \mathrm{~h}$ entre 10 a $100 \mathrm{mg} \mathrm{L}^{-1}$ y de $13.4 \mathrm{mg} \mathrm{L}^{-1}$ a 14 días de exposición. Pounds et al. (2008) encontraron en Planorbis carinatus Müller, 1774 valores menores a los observados en el presente estudio de $17.1 \mathrm{mg} \mathrm{L}-1$ a $72 \mathrm{~h}$ de exposición.

Con relación al paracetamol sobre $D$. magna fue observado un valor de $\mathrm{CL}_{50}$ a 96 h de $62.3 \mathrm{mg} \cdot \mathrm{L}^{-1}$ y es el único fármaco que en el presente estudio presentó un CR mayor a 1 debido a que el valor de efecto en $D$. magna fue mayor que el valor de exposición esperado. Touliabah et al. (2008) han observado efectos tóxicos del comunidades microbianas acuáticas (Maul et al., 2006). Robinson et al. (2005) al evaluar siete antibióticos floroquinolónicos sobre cinco organismos acuáticos Microcystis aeruginosa (Kutzing 1846) Lemmermann, 1907, Lemna minor Linnaeus, 1753, P. subcapitata, D. magna y Pimephales promelas Rafinesque 1820, encontraron que el ciprofloxacino solo en $M$. aeruginosa provocó riesgo en el agua superficial.

Tabla 4.- Cociente de riesgo estimado siete productos farmacéuticos y productos de cuidado personal (PPCPs).

\begin{tabular}{cccc}
\hline PPCPs & PEC $\left(\mathrm{mg} \cdot \mathrm{L}^{-1}\right)$ & PNEC $\left(\mathrm{mg} \cdot \mathrm{L}^{-1}\right)$ & Cociente de riesgo \\
\hline Amoxicilina & 0.0107 & 69.50 & $1.5 \times 10^{-4}$ \\
Ciprofloxacino & 0.00067 & 2.30 & $2.9 \times 10^{-4}$ \\
Diazepan & 0.0027 & 0.17 & 0.015 \\
Ibuprofeno & 0.10 & 1.75 & 0.057 \\
Paracetamol & 0.63 & 0.62 & 1.01 \\
Trimetoprima & 0.00017 & 2.96 & $5.74 \times 10^{-5}$ \\
Sulfametoxazol & 0.00674 & 14.80 & $4.5510^{-4}$ \\
\hline
\end{tabular}

PNEC $=\mathrm{CL}_{50} / 100$ (OECD, 1992). PEC para Amoxicilina, diazepan, ibuprofeno, sulfametoxazol y paracetamol fueron tomados de Kûmmerer (2004). PEC para Ciprofloxacino y Trimetoprima fueron tomados de Halling-Sórensen et al. (2000). 
Una evaluación de riesgos ecológicos de 13 antibióticos en Korea ha mostrado que la amoxicilina se encuentra entre los tres productos cuyo CR excede a 1 y por ende provoca riesgos de efectos tóxicos agudos (Lee et al., 2008). De igual manera una evaluación ambiental acuática de los farmacéuticos mas empleados en Inglaterra mostró que la amoxicilina y el paracetamol estuvieron entre los cuatro fármacos que excedieron el CR en 1 (Jones et al., 2002). En nuestro estudio solo el paracetamol por el CR, presentó riesgo en el ambiente acuático dulceacuícola; y por el contrario la amoxicilina presentó los valores de $\mathrm{CL}_{50}$ de menor toxicidad y de menor CR entre los fármacos ensayados (Tabla 3).

Con relación a los parámetros fisico-químicos las drogas poco metabolizadas y altamente excretadas en los sistemas animales son poco degradadas en el ambiente. Todos los fármacos evaluados en el presente estudio presentan bajos log Kow con valores menores a 5, lo que evidencian que no son muy lipofílicos (Jjemba, 2006). Para los fármacos evaluados, la solubilidad, el logKow, el pKa y el porcentaje excretado ampliamente usado en condiciones clínicas, no nos predicen por sí solos adecuadamente el riesgo ambiental al no relacionarse con diversos parámetros de toxicidad de los productos farmaceúticos. En el presente estudio no se encontró relación significativa entre el log $\mathrm{CL}_{50}$ de los PPCPs y sus log Kow, solubilidad y \% excretado del PPCPs. De igual forma, Jjemba (2006) para 81 PPCPs encontró que la solubilidad, el log Kow (indicador de lipofilidad del compuesto) y pKa no se encuentran relacionados, ni predicen el comportamiento de los PPCPs en el ambiente o su concentración en el ambiente. Se ha indicado que bajos valores de log Kow para los siete PPCPs como los mostrados en el presente trabajo, pudieran reducir su afinidad al suelo, sedimentos, metales y materia orgánica disuelta en el agua e incrementar la biodisponibilidad del compuesto en el ambiente; y por otro lado estos bajos valores podrían señalar una mayor polaridad de los siete PPCPs por aumento en la sustitución de oxígeno por halógenos en la relación $\mathrm{C} / \mathrm{O}$ y $\mathrm{H} / \mathrm{O}$ y facilitar su ingreso dentro de la célula. Sin embargo, sorprendentemente no se observó en el presente estudio que el log Kow de estos siete PPCPs se encontrará asociado con el log $\mathrm{CL}_{50}$ de los PPCPs. De igual forma la solubilidad y el \% excretado del PPCPs no se relacionaron con el log $\mathrm{CL}_{50}$ de los PPCPs posiblemente debido a que el ambiente es un sistema abierto y la solubilidad y degradabilidad dependen de una amplia variedad de factores como el $\mathrm{pH}$, metales y sales existentes, y temperaturas fluctuantes. Un bajo $\%$ excretado por una alta capacidad de metabolismo en un sistema animal pudiera señalar una mayor capacidad de degradación en el ambiente, y por ende una menor toxicidad. Sin embargo, el metabolito excretado no siempre es de menor toxicidad que la sustancia original, y en muchos casos es mucho más tóxico (Halling-Sørensen et al., 1998; Jjemba, 2006).

En este estudio se han empleado diferentes combinaciones de datos toxicológicos y fisicoquímicos para la evaluación del riesgo ambiental en el ambiente dulceacuícola empleando D. magna. Cooper et al. (2008) han encontrado diferentes componentes identificados como de alto riesgo según el método empleado. De igual forma se observan resultados no congruentes con la EP y CR para determinar el riesgo en el ambiente acuático dulceacuícola. Según la EP, los tres farmacéuticos con mayor riesgo son: Diazepan, trimetoprima y sulfametoxazol. En cambio, por el CR solo el paracetamol provoca riesgo. Nuestros resultados no excluyen el efecto potencial de toxicidad crónica en el ambiente de estos fármacos ensayados, como también ha sido señalado por Lee et al. (2008).

\section{Literatura citada.}

Backhaus T., Scholze M. \& Grimme L.H. 2000. The single substance and mixture toxicity of quinolones to the bioluminescent bacterium Vibrio fischeri. Aquatic Toxicology. 49: 49-61.

Bila D.M. \& Dezotti M. 2003. Fármacos no medio ambiente. Química Nova. 26: 523-530.

Brain R.A., Hanson M.L., Solomon K.R. \& Brooks B.W. 2008. Aquatic plants exposed to pharmaceuticals: effects and risks. Reviews of Environmental Contamination and Toxicology. 192: 67-115.

Burton G.A. Jr. \& Nordstrom J.E. 2004. An in situ toxicity identification evaluation method Part I: Laboratory validation. Environmental Toxicology and Chemistry. 23: 2844-2850.

Calvo J. \& Martínez L. 2009. Mecanismos de acción de los antimicrobianos. Enfermedades infecciosas y microbiología clínica. 27: 44-52.

Carabaño A.I., Jiménez L.I., López. C. P.M., Calvo G.I., Pello L.A.M., Balugo B.P., Baro F.M. \& Ruiz C. J. 2005. Eficacia de ibuprofeno y paracetamol como antitérmicos. Anales Pediátricos (Barcelona). 62: 117122

Castillo G. 2004. Ensayos toxicológicos y métodos de evaluación de calidad de aguas. Estandarización, intercalibración, resultados y aplicaciones. IMTA. México.

Castiglioni S., Fanelli R., Calamari D., Bagnati R. \& Zuccato E. 2004. Methodology approaches for studying pharmaceuticals in the environment by comparing predicted and measured concentrations in the river Po, Italy. Regulatory Toxicology and Pharmacology. 39: 25-32.

Champan P.M. 2006. Emerging substances- emerging problems? Environmental Toxicology and Chemistry. 25: 1445-1447.

Choi K., Kim Y., Jung J., Kim M.H., Kim C.S., Kim N.H. \& Park J. 2008. Occurrence and ecological risks of roxithromycin, trimethoprim, and chloramphenicol in the Han river, Korea. Environmental Toxicology and Chemistry. 27: 711-719.

Cooper E.R., Siewicki T.C. \& Phillips K. 2008. Preliminary risk assessment database and risk ranking of 
pharmaceuticals in the environment. Science of The Total Environment. 398: 26-33.

Daughton C.G. \& Ternes T.A. 1999. Pharmaceutical and personal care products in the environment: agents of subtle change?. Environmental Health Perspectives. 107: 907-938.

De Liguoro M., Fioretto B., Poltronieri C. \& Gallina G. 2009. The toxicity of sulfamethazine to Daphnia magna and its additivity to other veterinary sulfonamides and trimethoprim. Chemosphere. 75:1519-1524.

Filho R.W.R., Barreiro J.C., Vieira E.M. \& Cass Q.B. 2007. Fármacos, ETEs e corpos hídricos. Revista Ambiente \& Agua- An International Journal of Applied Science. 2: 54-61.

Halling-Sørensen B., Nor Nielsen S., Lanzky P.P.F., Ingerslev F., Lützhøft H.C. \& Jorgensen S.E. 1998. Occurrence, fate, and effects of pharmaceutical substances in the environment- A review. Chemosphere. 36: 357-393.

Halling-Sørensen B., Loteen-Lûtzhoft H.C., Andersen H.R. \& Ingerslev F. 2000. Environmental risk assessment of antibiotics: comparison of mecillinam, trimethoprim and ciprofloxacin. Journal of Antimicrobial Chemotherapy. 46, Suppl. SI: 53-58.

Heckmann L.H. 2007. Chronic toxicity of ibuprofen to Daphnia magna: effects on life history traits and population dynamics. Toxicology letters. 172: 137-145.

Jjemba P.K. 2006. Excretion and ecotoxicity of pharmaceutical and personal care products in the environment. Ecotoxicology and Environmental Safety. 63: $113-130$.

Jones O.A.H., Voulvoulis J.N. \& Lester J.N. 2002. Aquatic environmental assessment fo the top 25 english prescription pharmaceuticals. Water Research. 36: 5013-5022.

Källqvist T., Grung M. \& Tollefsen K.E. 2006. Chronic toxicity of 2,4,2', 4'-Tetrabromodiphenil ether on the marine alga Skeletonema costatum and the crustacean Daphnia magna. Environmental Toxicology and Chemistry. 25: 1657-1662.

Kim Y., Choi K., Jung J., Park S., Kim P.G. \& Park J. 2007. Aquatic toxicity of acetaminophen, carbamazepine, cinetidine, ditiazem and six major sulfonamides, and their potential ecological risks in Korea. Environmental International. 33: 370-375.

Klüttgen B., Dülmer U., Engels M. \& Ratte H.T. 1994. $\mathrm{ADaM}$, an artificial freshwater for culture of zooplankton. Water Research. 28: 743-746.

Kratz W. 2008. Ecotoxicological risk of human pharmaceuticals in Brandenburg surface waters?. Pp. 379-389. In: Schmidt M., Glasson J., Emmelin L. \& Helbron H. (eds.). Standards and thresholds for impact assessment. Environmental Protection in the European Union. Vol. 3. Ed. Springer-Verlag. Berlin.

Kümmerer K. 2003. Significance of antibiotics in the environment. Journal of Antimicrobial Chemotherapy. 52: 5-7.

Kümmerer K. 2004. Pharmaceuticals in the environment: sources, fate, effects and risks. $2^{\text {th }}$ Ed. Springer.

Lalumera G.M., Calamari D., Galli P., Castiglioni S., Crosa G. \& Fanelli R. 2004. Preliminary investigation on the environment occurence and effects of antibiotics used in aquaculture in Italy. Chemosphere. 54: 661-668.
Lee Y.L., Lee S.E., Lee D.S. \& Kim Y.H. 2008. Risk assessment of human antibiotics in korean aquatic environment. Environmental Toxicology and Pharmacology. 26: 216-221.

Lützhøft H.C.H., Halling-Sørensen B. \& Jørgensen S.E. 1999. Algal toxicity of antibacterial agents applied in Danish fish farming. Archives of Environmental Contamination and Toxicology. 36: 1-6.

Maul J.D., Schuler L.J., Belden J.B., Whiles M.R. \& Lydi M.J. 2006. Effects of the antibiotic ciprofloxacin on stream microbial communities and detritivorous macroinvertebrates. Environmental Toxicology and Chemistry. 25: 1598-1606.

OECD (Organization for Economic Cooperation and Development). 1992. Report of the OECD workshop on the Extrapolation of Laboratory Aquatic Toxicity Data to the Real Environment. Environment Monograph 59, OECD, Paris.

Park S. \& Choi K. 2008. Hazard assessment of commonly used agricultural antibiotics on aquatic ecosystems. Ecotoxicology. 17: 526-538.

Pounds N., MacLean S., Webley M., Pascoe D. \& Hutchinson T. 2008. Acute and chronic effects of ibuprofeno in the mollusc Planorbis carinatus (Gastropoda: Planorbidae). Ecotoxicology and environmental Safety. 70: 47-52.

Reynaldi S., Duquesne S., Jung K. \& Liess M. 2006. Linking feeding activity and maturation of Daphnia magna following short-term exposure to fenvalerate. Environmental Toxicology and Chemistry. 25: 18311835.

Robinson A.A., Belden J.B. \& Lydi M.J. 2005. Toxicity of fluoroquinolone antibiotics to aquatic organisms. Environmental toxicology and Chemistry. 24: 423-430.

Siemens J., Huschek G., Siebe C. \& Kaupenjohann M. 2008. Concentration and mobility of human pharmaceuticals in the world`s largest wastewater irrigation system, Mexico City-Mezquital Valley. Water Research. 42: 2124-2134.

Straub J.O. 2008. Deterministic and probabilistic environmental risk assessment for diazepam. Pp. 343383. In. Kümmerer K. Pharmaceuticals in the Environment Sources, Fate, Effects and Risks. $3^{\text {th }}$ Ed. Springer Berlin Heidelberg.

Tonkopii V., Zagrebin A. \& Iofina I. 2008. Bioidentification of xenobiotics as a basis of water management. In: pp. 349-353. Gönenç E., Vadineanu A, Wolflin J.P. \& Russo R.C. (Eds.). Sustainable use and development of watersheds. Springer Science + Business Media B.V. Netherlands.

Touliabah H.E., El-Bassat R.A., El-Shimy A. \& Harisa G.I. 2008. Oxidative stress of plankton community and some isolated species during paracetamol toxicity test. Journal of Biological Sciences. 8: 13-23.

Woodward K.N. 2006. Veterinary pharmacovigilance. Part 3. Adverse effects of veterinary medicinal products in animals and on the environment. Journal of Veterinary Pharmacology Therapethics. 28: 171-184.

Wong C.K. \& Pak A.P. 2004. Acute and subchronic toxicity of the heavy metals copper, chromium, nickel, and zinc, individually and in mixture, to the freshwater copepod Mesocyclops pehpeiensis. Bulletin of Environmental Contamination and Toxicology. 73:190196. 
Yang L.H., Ying G.G., Su H.C., Stauber J., Adams M. \& Binet M. 2008. Growth-inhibiting effects of twelve antibacterial agents and their mixtures on the freshwater microalga Pseudokirchneriella subcapitata. Environmental Toxicology and Chemistry. 27: 12011208.
Zuccato E., Castiglioni S., Fanelli R., Reitano G., Bagnati R., Chiabrando C., Pomati F., Rossetti C. \& Calamari D. 2006. Pharmaceuticals in the environment in Italy: causes, occurrence, effects and control. Environmental science and pollution research internacional. 13: 15-21.

${ }^{1}$ Museo de Historia Natural. Facultad de Ciencias Biológicas. Universidad Ricardo Palma. Av. Benavides 5440, Santiago de Surco, Lima, Perú.

2 Laboratorio de Ecofisiología Animal. Facultad de Ciencias Naturales y Matemática. Universidad Nacional Federico Villarreal. Email: joseiannacone@gmail.com.

${ }^{3}$ Laboratorio de Ecofisiología Animal. Facultad de Ciencias Naturales y Matemática. Universidad Nacional Federico Villarreal. 